\title{
EXISTENCE OF SUM AND PRODUCT INTEGRALS
}

\author{
BY
}

\author{
JON C. HELTON
}

This paper is dedicated to Professor H. S. Wall.

ABSTRACT. Functions are from $R \times R$ to $R$, where $R$ represents the set of real numbers. If $c$ is a number and either (1) $\int_{a}^{b} G^{2}$ exists and $\int_{a}^{b} G$ exists, (2) $\int_{a}^{b} G$ exists and $a^{\Pi^{b}}(1+G)$ exists and is not zero or (3) each of $a^{\Pi^{b}}(1+G)$ and $\Pi^{b}(1-G)$ exists and is not zero, then $\int_{a}^{b} c G$ exists, $\int_{a}^{b}\left|c G-\int c G\right|=0, x^{\Pi^{y}}(1+c G)$ exists for $a \leq x<y \leq b$ and $\int_{a}^{b}|1+c G-\Pi(1+c G)|=0$. Furthermore, if $H$ is a function such that $\lim _{x \rightarrow p^{-}} H(x, p), \lim _{x \rightarrow p^{+}} H(p, x), \lim _{x, y \rightarrow p^{-}} H(x, y)$ and $\lim _{x, y \rightarrow p^{+}} H(x, y)$ exist for each $p \in[a, b], n \geq 2$ is an integer, and $G$ satisfies either (1), (2) or (3) of the above, then $\int_{a}^{b} H G^{n}$ exists, $\int_{a}^{b}\left|H G^{n}-\int H G^{n}\right|=0,{ }_{x}^{\Pi^{y}}\left(1+H G^{n}\right)$ exists for $a \leq x<y \leq b$ and $\int_{a}^{b}\left|1+H G^{n}-\mathbf{n}\left(1+H G^{n}\right)\right|=0$.

All integrals and definitions are of the subdivision-refinement type, and functions are from $R \times R$ to $R$, where $R$ represents the set of real numbers. Furthermore, functions are assumed to be defined only for elements $\{x, y\}$ of $R \times R$ such that $x \leq y$, and $G(x, x)=0$. The statements that $G$ is bounded, $G \in O P^{\circ}$, $G \in O Q^{\circ}$ and $G \in O B^{\circ}$ on $[a, b]$ mean that there exist a subdivision $D$ of $[a, b]$ and positive numbers $B$ and $c$ such that if $J=\left\{x_{q}\right\}_{0}^{n}$ is a refinement of $D$, then

(1) $|G(u)|<B$ for $u \in J(I)$,

(2) $\left|\Pi_{i}^{j}\left(1+G_{q}\right)\right|<B$ for $1 \leq i \leq j \leq n$,

(3) $\left|\Pi_{i}^{j}\left(1+G_{q}\right)\right|>c$ for $1 \leq i \leq j \leq n$, and

(4) $\Sigma_{J(I)}|G|<B$,

respectively, where $G_{q}=G\left(x_{q-1}, x_{q}\right)$ and $J(I)=\left\{\left[x_{q-1}, x_{q}\right]\right\}_{1}^{n}$. Similarly, statements of the form $G>b$ should be interpreted in terms of subdivisions and refinements. The symbols $G\left(p, p^{+}\right), G\left(p^{-}, p\right), G\left(p^{+}, p^{+}\right)$and $G\left(p^{-}, p^{-}\right)$are used to denote $\lim _{x \rightarrow p^{+}} G(p, x), \lim _{x \rightarrow p^{-}} G(x, p), \lim _{x, y \rightarrow p^{+}} G(x, y)$, and $\lim _{x, y \rightarrow p^{-}} G(x, y)$, respectively. Further, $G \in O L^{\circ}$ on $[a, b]$ only if $G\left(p, p^{+}\right)$, $G\left(p^{-}, p\right), G\left(p^{+}, p^{+}\right)$and $G\left(p^{-}, p^{-}\right)$exist for each $p \in[a, b]$, and $G \in O L^{14}$ on $[a, b]$ only if $G \in O L^{\circ}$ on $[a, b]$ and $G\left(p^{+}, p^{+}\right)=G\left(p^{-}, p^{-}\right)=0$ for each $p \epsilon$ $[a, b]$. For convenience in notation, when we consider a function $G$ defined only

Presented to the Society, January 28, 1973; received by the editors July 19, 1972. AMS (MOS) subject classifications (1970). Primary 26A39, 26A42, 28A25.

Key words and phrases. Sum integral, product integral, subdivision-refinement integral, existence, interdependency, interval function. 
on intervals $[x, y]$ such that $a \leq x<y \leq b$, we adopt the convention that

$$
G\left(a^{-}, a^{-}\right)=G\left(a^{-}, a\right)=G\left(b^{+}, b^{+}\right)=G\left(b, b^{+}\right)=0 .
$$

Also, $G \in O A^{\circ}$ on $[a, b]$ only if $\int_{a}^{b} G$ exists and $\int_{a}^{b}\left|G-\int G\right|=0$, and $G \in O M^{\circ}$ on $[a, b]$ only if $\Pi^{y}(1+G)$ exists for $a \leq x<y \leq b$ and $\int_{a}^{b}|1+G-\Pi(1+G)|$ $=0$. The sources of these definitions are [3, p. 299], [4, p. 493], [5] and [7].

Lemma 1.1. If $\int_{a}^{b} G^{2}$ exists and $\int_{a}^{b} G$ exists, then $G \in O L^{14}$ on $[a, b]$.

The proof of Lemma 1.1 is straightforward, and therefore we omit it.

Lemma 1.2. If $\beta>0,|G|<1-\beta$ on $[a, b], \int_{a}^{b} G^{2}$ exists and $\int_{a}^{b} G$ exists, then $\Pi_{a} \Pi^{b}(1+G)$ exists and is not zero $[6$, Theorem 5$]$.

Lemma 1.3. If $G$ is bounded on $[a, b]$ and $\Pi^{b}(1+G)$ exists and is not zero, then $G \in O P^{\circ}$ and $O Q^{\circ}$ on $[a, b][7$, Theorem 2].

Lemma 1.4. If $G$ is bounded on $[a, b]$ and $\Pi^{b}(1+G)$ exists and is not zero, then $G \in O M^{\circ}$ on $[a, b]$.

Indication of Proof. This lemma follows from Lemma 1.3 and a result of B. W. Helton [3, Theorem 4.2, p. 305].

Lemma 1.5. If $\int_{a}^{b} G$ exists, then $G \in O A^{\circ}$ on $[a, b]$.

This result is due to A. Kolmogoroff [8, p. 669]. The reader is also referred to results by W. D. L. Appling [1, Theorems 1, 2, p. 155] and B. W. Helton [3, Theorem 4.1, p. 304].

Lemma 1.6. If $E=\left\{p_{i}\right\}_{1}^{r}$ is a set of distinct points from $[a, b]$ and $F, G$ and $H$ are functions defined on $[a, b]$ sucb that

(1) $G \in O L^{\circ}$ on $[a, b]$ and $\int_{a}^{b} G$ exists,

(2) if $p \in E$, then $H\left(p, p^{+}\right)$and $H\left(p^{-}, p\right)$ exist, and

(3) if $a \leq x<y \leq b$, then $F(x, y)=G(x, y)$ if $x \notin E$ and $y \notin E$ and $F(x, y)$ $=H(x, y)$ if $x \in E$ or $y \in E$, then $\int_{a}^{b} F$ exists and is

$$
\int_{a}^{b} G+\sum_{p \in E}\left[H\left(p, p^{+}\right)+H\left(p^{-}, p\right)-G\left(p, p^{+}\right)-G\left(p^{-}, p\right)\right]
$$

The proof of Lemma 1.6 is straightforward, and therefore we omit it. 
Lemma 1.7. If $E=\left\{p_{i}\right\}_{1}^{r}$ is a set of distinct points from $[a, b]$ and $F, G$ and $H$ are functions on $[a, b]$ sucb that

(1) $G \in O L^{\circ}$ on $[a, b]$ and $\Pi^{b}(1+G)$ exists and is not zero,

(2) if $p \in E$, then $H\left(p, p^{+}\right)$and $H\left(p^{-}, p\right)$ exist, and

(3) if $a \leq x<y \leq b$, then $F(x, y)=G(x, y)$ if $x \notin E$ and $y \notin E$ and $F(x, y)$ $=H(x, y)$ if $x \in E$ or $y \in E$,

then $\Pi^{b}(1+F)$ exists and is

$$
\begin{aligned}
\left\{a^{b}(1+G)\right\} & \cdot\left\{\prod_{p \in E}\left[1+H\left(p, p^{+}\right)\right][1+H(p-, p)]\right\} \\
& \cdot\left\{\prod_{p \in E}\left[1+G\left(p, p^{+}\right)\right]\left[1+G\left(p^{-}, p\right)\right]\right\}^{-1} .
\end{aligned}
$$

Furtbermore, $F \in O M^{\circ}$ on $[a, b]$.

Proof. We first show that ${ }_{a} \Pi^{b}(1+F)$ exists and is $P$, where

$$
\begin{aligned}
P & =\left[\prod^{b}(1+G)\right]\left[P_{1}\right]\left[P_{2}\right]^{-1}, \\
P_{1} & =\prod_{p \in E}\left[1+H\left(p, p^{+}\right)\right]\left[1+H\left(p^{-}, p\right)\right]
\end{aligned}
$$

and

$$
P_{2}=\prod_{p \in E}\left[1+G\left(p, p^{+}\right)\right]\left[1+G\left(p^{-}, p\right)\right]
$$

Since $G \in O L^{\circ}$ on $[a, b]$, it follows from the covering theorem that $G$ is bounded on $[a, b]$. Hence, it follows from Lemma 1.3 that $G \in O P^{\circ}$ and $O Q^{\circ}$ on $[a, b]$. Let $\epsilon>0$. There exist a subdivision $D_{1}$ of $[a, b]$ and a number $B>1$ such that if $J=\left\{x_{q}\right\}_{0}^{n}$ is a refinement of $D_{1}$, then

(1) $\left|\Pi_{i}^{i}\left(1+G_{q}\right)\right|<B$ for $1 \leq i \leq j \leq n$,

(2) $\left|P_{1}\right|<B$ and $\left|\left[P_{2}\right]^{-1}\right|<B$, and

(3) $\left|\Pi_{a} \Pi^{b}(1+G)-\Pi_{J(I)}(1+G)\right|<\epsilon\left(3 B^{2}\right)^{-1}$.

Let $\delta$ be a positive number such that if

$$
\left.p_{i} \stackrel{a}{-} \delta\right\}<x_{i}<p_{i}<y_{i}<\left\{p_{i} \stackrel{b}{+} \delta\right.
$$

for $1 \leq i \leq r$, then 


$$
\left|1-\left\{P_{2}\right\}^{-1}\left\{\prod_{i=1}^{r}\left[1+G\left(x_{i}, p_{i}\right)\right]\left[1+G\left(p_{i}, y_{i}\right)\right]\right\}\right|<\epsilon\left(3 B^{2 r+1}\right)^{-1}
$$

and

$$
\left|P_{1}-\prod_{i=1}^{r}\left[1+H\left(x_{i}, p_{i}\right)\right]\left[1+H\left(p_{i}, y_{i}\right)\right]\right|<\epsilon\left(3 B^{2 r}\right)^{-1}
$$

Let $D$ denote the subdivision of $[a, b]$ consisting of

$$
D_{1} \cup\left\{p_{i}-\delta\right\}_{1}^{r} \cup\left\{p_{i}+\delta\right\}_{1}^{r} \cup\left\{1 / 2\left(p_{i}+p_{i+1}\right)\right\}_{1}^{r-1} \cup E
$$

less any elements which are not in $[a, b]$. Suppose $J$ is a refinement of $D$. Let $K(I)$ be the subset of $J(I)$ such that $u \in K(I)$ only if neither end point of $u$ belongs to $E$. Note that no interval in $J(I)$ can have elements of $E$ at both end points. Let $L(I)=J(I)-K(I)$. Thus,

$$
\begin{aligned}
\left|\prod_{J(I)}(1+F)-P\right| \leq & \left|\prod_{J(I)}(1+F)-\left[\prod_{J(I)}(1+G)\right]\left[P_{1}\right]\left[P_{2}\right]-1\right| \\
& +\left|\left[\prod_{J(I)}(1+G)\right]\left[P_{1}\right]\left[P_{2}\right]^{-1}-P\right| \\
& <\left|\prod_{K(I)}(1+G)\right|\left|\prod_{L(I)}(1+F)-\left[\prod_{L(I)}(1+G)\right]\left[P_{1}\right]\left[P_{2}\right]^{-1}\right|+\epsilon\left(3 B^{2}\right)^{-1} B^{2} \\
\leq & B^{2 r}\left|\prod_{L(I)}(1+F)-\left[\prod_{L(I)}(1+G)\right]\left[P_{1}\right]\left[P_{2}\right]^{-1}\right|+\frac{\epsilon}{3} \\
\leq & B^{2 r}\left|\prod_{L(I)}(1+F)-P_{1}\right|+B^{2 r}\left|P_{1}\right|\left|1-\left[\prod_{L(I)}(1+G)\right]\left[P_{2}\right]^{-1}\right|+\frac{\epsilon}{3} \\
& <\epsilon\left(3 B^{2 r}\right)^{-1} B^{2 r}+\epsilon\left(3 B^{2 r+1}\right)^{-1} B^{2 r+1}+\epsilon / 3=\epsilon .
\end{aligned}
$$

Therefore, $a^{b}(1+F)$ exists and is $P$.

We now show that $F \in O M^{\circ}$ on $[a, b]$. Since it can be shown that $\Pi_{x}^{y}(1+F)$ exists for $a \leq x<y \leq b$ by an argument similar to the one used in the preceding paragraph, it is only necessary to show that

$$
\int_{a}^{b}|1+F-\Pi(1+F)|=0
$$


Let $\epsilon>0$. As noted in the previous paragraph, $G \in O P^{\circ}$ and $O Q^{\circ}$ on $[a, b]$. Hence, Lemma 1.4 implies that $G \in O M^{\circ}$ on $[a, b]$. There exist a subdivision $D_{1}$ of $[a, b]$ and a number $B>1$ such that if $\left\{x_{q}\right\}_{0}^{n}$ is a refinement of $D_{1}$, then

(1) $\left|\Pi_{i}^{j}\left(1+G_{q}\right)\right|<B$ for $1 \leq i \leq j \leq n$,

(2) $\left|1+F_{q}\right|<B$ and $\left|1+G_{q}\right|>1 / B$ for $1 \leq q \leq n$, and

(3) $\sum_{q=1}^{n}\left|1+G_{q}-\Pi_{J_{q}(I)}(1+G)\right|<\epsilon\left(5 B^{2}\right)^{-1}$, where $J_{q}$ is a subdivision of $\left[x_{q-1}, x_{q}\right]$ for $1 \leq q \leq n$.

Let $\delta$ be a positive number such that if $1 \leq i \leq r$ and

$$
\left.p_{i} \stackrel{a}{-} \delta\right\}<x_{i}^{\prime}<x_{i}<p_{i}<y_{i}^{\prime}<y_{i}<\left\{p_{i} \stackrel{b}{+} \delta,\right.
$$

then

(1) $\left|F\left(x_{i}, p_{i}\right)-F\left(x_{i}^{\prime}, p_{i}\right)\right|<\epsilon(10 r)^{-1}$,

(2) $\left|F\left(p_{i}, y_{i}\right)-F\left(p_{i}, y_{i}^{\prime}\right)\right|<\epsilon(10 r)^{-1}$,

(3) $\left|G\left(x_{i}, p_{i}\right)-G\left(x_{i}^{\prime}, p_{i}\right)\right|<\epsilon\left(10 r B^{3}\right)^{-1}$, and

(4) $\left|G\left(p_{i}, y_{i}\right)-G\left(p_{i}, y_{i}^{\prime}\right)\right|<\epsilon\left(10 r B^{3}\right)^{-1}$.

Let $D$ denote the subdivision of $[a, b]$ consisting of

$$
D_{1} \cup\left\{p_{i}-\delta\right\}_{1}^{r} \cup\left\{p_{i}+\delta\right\}_{1}^{r} \cup\left\{1 / 2\left(p_{i}+p_{i+1}\right)\right\}_{1}^{r-1} \cup E
$$

less any elements which are not in $[a, b]$.

Suppose $J=\left\{x_{q}\right\}_{0}^{n}$ is a refinement of $D$. For $1 \leq q \leq n$, let $J_{q}=\left\{x_{q, i}\right\}_{0}^{n(q)}$ be a subdivision of $\left[x_{q-1}, x_{q}\right]$ such that

$$
\left|\prod_{q-1} \prod^{x_{q}}(1+F)-\prod_{J_{q}(l)}(1+F)\right|<\frac{\epsilon}{5 n} .
$$

Also, for $1 \leq q \leq n$, suppose

(1) $q \in U$ only if $\left[x_{q-1}, x_{q}\right]$ does not have a point of $E$ as an end point,

(2) $q \in V(1)$ only if $x_{q} \in E$, and

(3) $q \in V(2)$ only if $x_{q-1} \in E$.

Note that $D$ is defined so that $q$ cannot belong to both $V(1)$ and $V(2)$. For $q \in$ $V(1)$, let
(1) $K_{q}=\left\{x_{q, i}\right\}_{0}^{n(q)-1}$,
(2) $F_{q}^{\prime}=F\left(x_{q, n(q)-1}, x_{q, n(q)}\right)$, and
(3) $G_{q}^{\prime}=G\left(x_{q, n(q)-1}, x_{q, n(q)}\right)$,

and for $q \in V(2)$, let

(1) $K_{q}=\left\{x_{q, i}\right\}_{1}^{n(q)}$,

(2) $F_{q}^{\prime}=F\left(x_{q, 0}, x_{q, 1}\right)$, and

(3) $G_{q}^{\prime}=G\left(x_{q, 0}, x_{q, 1}\right)$.

If $q \leqslant V(1)$ or $V(2)$, then $\Pi_{J_{q}(I)}(1+F)=\left(1+F_{q}^{\prime}\right) \Pi_{K_{q}(I)}(1+G)$ and $\Pi_{J_{q}(I)}(1+G)=\left(1+G_{q}^{\prime}\right) \Pi_{K_{q}(I)}(1+G)$. Thus, 


$$
\begin{aligned}
& \sum_{q=1}^{n}\left|1+F_{q}-x_{q-1} \prod^{x_{q}}(1+F)\right|<\sum_{q=1}^{n}\left|1+F_{q}-\prod_{J_{q}(I)}(1+F)\right|+\frac{\epsilon}{5} \\
& =\sum_{q \in U}\left|1+G_{q}-\prod_{J_{q}(I)}(1+G)\right|+\sum_{i=1}^{2} \sum_{q \in V(i)}\left|1+F_{q}-\prod_{J_{q}(I)}(1+F)\right|+\frac{\epsilon}{5} \\
& <\frac{\epsilon}{5}+\sum_{i=1}^{2} \sum_{q \in V(i)}\left|1+F_{q}-\left(1+F_{q}^{\prime}\right) \prod_{K_{q}(I)}(1+G)\right|+\frac{\epsilon}{5} \\
& \leq \sum_{i=1}^{2} \sum_{q \in V(i)}\left|1+F_{q}^{\prime}\right|\left|1-\prod_{K_{q}(I)}(1+G)\right|+\sum_{i=1}^{2} \sum_{q \in V(i)}\left|F_{q}-F_{q}^{\prime}\right|+\frac{2 \epsilon}{5} \\
& <B \sum_{i=1}^{2} \sum_{q \in V(i)}\left|1-\prod_{K_{q}(I)}(1+G)\right|+[2 r]\left[\epsilon(10 r)^{-1}\right]+\frac{2 \epsilon}{5} \\
& =B \sum_{i=1}^{2} \sum_{q \in V(i)}\left|\left(1+G_{q}\right)^{-1}\right|\left|1+G_{q}-\left(1+G_{q}\right) \prod_{K_{q}(I)}(1+G)\right|+\frac{3 \epsilon}{5} \\
& \leq B^{2} \sum_{i=1}^{2} \sum_{q \in V(i)}\left|1+G_{q}-\left(1+G_{q}\right) \prod_{K_{q}(I)}(1+G)\right|+\frac{3 \epsilon}{5} \\
& \leq B^{2} \sum_{i=1}^{2} \sum_{q \in V(i)}\left|1+G_{q}-\prod_{J_{q}(I)}(1+G)\right| \\
& +B^{2} \sum_{i=1}^{2} \sum_{q \in V(i)}\left|G_{q}-G_{q}^{\prime}\right|\left|\prod_{K_{q}(I)}(1+G)\right|+\frac{3 \epsilon}{5} \\
& <B^{2}\left[\epsilon\left(5 B^{2}\right)^{-1}\right]+\left[2 r B^{3}\right]\left[\epsilon\left(10 r B^{3}\right)^{-1}\right]+\frac{3 \epsilon}{5}=\epsilon \text {. }
\end{aligned}
$$

Therefore, $F \in O \dot{M}^{\circ}$ on $[a, b]$.

Theorem 1. If $\int_{a}^{b} G^{2}$ exists, $\int_{a}^{b} G$ exists and $c$ is a number, then $c G \in O M^{\circ}$ and $O A^{\circ}$ on $[a, b]$.

Proof. Since it follows from Lemma 1.5 that $c G \in O A^{\circ}$ on $[a, b]$, we need only show that $c G \in O M^{\circ}$ on $[a, b]$. Since $G \in O L^{14}$ on $[a, b]$ [Lemma 1.1], there exists a subdivision $D=\left\{x_{q}\right\}_{0}^{n}$ of $[a, b]$ such that if $1 \leq q \leq n$ and $x_{q-1}$ $<x<y<x_{q}$, then $|c G(x, y)|<1 / 2$. Let $F$ be the function such that

(1) $F(x, y)=c G(x, y)$ if $x \notin D$ and $y \notin D$, and

(2) $F(x, y)=0$ if $x \in D$ or $y \in D$.

Thus, $|F|<1 / 2$, and Lemma 1.6 implies that $\int_{a}^{b} F^{2}$ exists and $\int_{a}^{b} F$ exists. Therefore, $a^{b}(1+F)$ exists and is not zero by Lemma 1.2, and hence, Lemma 1.7 implies that $c G \in O M^{\circ}$ on $[a, b]$. 
Lemma 2.1. If $G \in O M^{\circ}$ or $O A^{\circ}$ on $[a, b], G \in O B^{\circ}$ on $[a, b]$ and $H \in O L^{\circ}$ on $[a, b]$, then $H G \in O M^{\circ}$ and $O A^{\circ}$ on $[a, b][4$, Theorem 2, p. 494], [3, Theorem $3.4, p .301]$.

Theorem 2. If $\int_{a}^{b} G^{2}$ exists, $\int_{a}^{b} G$ exists, $H \in O L^{\circ}$ on $[a, b]$ and $n \geq 2$ is an integer, then $H G^{n} \in O M^{\circ}$ and $O A^{\circ}$ on $[a, b]$.

Proof. Since $G \in O L^{14}$ on $[a, b]$ [Lemma 1.1], it follows that $H G^{n-2} \in O L^{\circ}$ on $[a, b]$. Therefore, since $G^{2} \in O A^{\circ}$ on $[a, b]$ [Lemma 1.5] and $G^{2} \in O B^{\circ}$ on $[a, b]$, it follows from Lemma 2.1 that $H G^{n} \in O M^{\circ}$ and $O A^{\circ}$ on $[a, b]$.

Theorem 2 is not true for $n=1$. The author [5, Theorem 10] has shown that for $\int_{a}^{b} H G$ to exist for every $H \in O L^{\circ}$ on $[a, b]$ it is necessary that $G \in O B^{\circ}$ on $[a, b]$. However, Davis and Chatfield [2, p. 747] define a function $G$ such that $\int_{a}^{b} G^{2}$ exists, $\int_{a}^{b} G$ exists and $G \notin O B^{\circ}$ on $[a, b]$.

Lemma 3.1. If $\Pi^{b}(1+G)$ exists and is not zero and $\int_{a}^{b} G$ exists, then $G \in O L^{14}$ on $[a, b][6$, Theorem 9].

The conclusion of Theorem $9[6]$ states that $G \in O L^{\circ}$ on $[a, b]$. However, the argument used to establish this also establishes that $G \in O L^{14}$ on $[a, b]$.

Lemma 3.2. If $\int_{a}^{b} H G$ exists, $G \geq 0$ on $[a, b], H \in O L^{\circ}$ on $[a, b]$ and $H$ is bounded away from zero on $[a, b]$, then $\int_{a}^{b} G$ exists.

Proof. There exists a subdivision $\left\{x_{q}\right\}_{0}^{n}$ of $[a, b]$ such that if $1 \leq q \leq n$, then $H$ does not change sign on $\left(x_{q-1}, x_{q}\right)$. Hence, $H G \in O B^{\circ}$ on $\left[x_{q-1}, x_{q}\right]$, and thus, $H G \in O B^{\circ}$ on $[a, b]$. Therefore, since $H^{-1} \in O L^{\circ}$ on $[a, b]$ and $H G \epsilon$ $O A^{\circ}$ on $[a, b]$ [Lemma 1.5], it follows from Lemma 2.1 that $\int_{a}^{b} G$ exists.

Lemma 3.3. If $\Pi^{b}(1+G)$ exists and is not zero and $G>-1$ on $[a, b]$, then $\int_{a}^{b} \ln (1+G)$ exists [6, Theorem 4].

Lemma 3.4. If $\Pi^{b}(1+G)$ exists and is not zero and $\int_{a}^{b} G$ exists, then $\int_{a}^{b} G^{2}$ exists.

Proof. Since $G \in O L^{14}$ on $[a, b]$ [Lemma 3.1], there exists a subdivision $D=\left\{x_{q}\right\}_{0}^{n}$ of $[a, b]$ such that if $1 \leq q \leq n$ and $x_{q-1}<x<y<x_{q}$, then $|G(x, y)|<$ 1/2. Let $F$ be the function such that

(1) $F(x, y)=G(x, y)$ if $x \notin D$ and $y \notin D$, and

(2) $F(x, y)=0$ if $x \in D$ or $y \in D$.

Therefore, $\Pi^{b}(1+F)$ exists and is not zero by Lemma 1.7, and $\int_{a}^{b} F$ exists by Lemma 1.6. Thus, from Lemma 3.3, $\int_{a}^{b} \ln (1+F)=\int_{a}^{b} \sum_{n=1}^{\infty}(-1)^{n-1} F^{n} / n$ exists, 
and hence,

$$
\int_{a}^{b} \sum_{n=2}^{\infty}(-1)^{n-1} \frac{F^{n}}{n}=\int_{a}^{b} F^{2}\left[-\frac{1}{2}+\sum_{n=3}^{\infty}(-1)^{n-1} \frac{F^{n-2}}{n}\right]
$$

exists. Thus, since $-1 / 2+\sum_{n=3}^{\infty}(-1)^{n-1} F^{n-2} / n$ is in $O L^{\circ}$ on $[a, b]$ and is bounded away from zero on $[a, b]$, Lemma 3.2 implies that $\int_{a}^{b} F^{2}$ exists. Therefore, $\int_{a}^{b} G^{2}$ exists by Lemma 1.6.

Theorem 3. If $\Pi^{b}(1+G)$ exists and is not zero, $\int_{a}^{b} G$ exists and $c$ is a number, then $c G \in O M^{\circ}$ and $O A^{\circ}$ on $[a, b]$.

Proof. It follows from Lemma 3.4 that $\int_{a}^{b} G^{2}$ exists. Therefore, Theorem 3 follows from Theorem 1 .

Theorem 4. If $\Pi^{b}(1+G)$ exists and is not zero, $\int_{a}^{b} G$ exists, $H \in O L^{\circ}$ on $[a, b]$ and $n \geq 2$ is an integer, then $H G^{n} \in O M^{\circ}$ and $O A^{\circ}$ on $[a, b]$.

Proof. It follows from Lemma 3.4 that $\int_{a}^{b} G^{2}$ exists. Therefore, Theorem 4 follows from Theorem 2 .

Lemma 5.1. If eacb of $\Pi^{b}(1+G)$ and $\Pi^{b}(1-G)$ exists and is not zero, then $G$ is bounded on $[a, b][7$, Lemma 6.1].

Lemma 5.2. If eacb of $a^{b}(1+G)$ and $\Pi^{b}(1-G)$ exists and is not zero, then $G \in O L^{14}$ on $[a, b]$.

Proof. Let $p \in(a, b]$. It follows from Lemmas 5.1 and 1.3 that $G \in O Q^{\circ}$ and $-G \in O Q^{\circ}$ on $[a, b]$. By applying this result and the Cauchy criterion for product integrals, we have that

(1) $0=\lim _{x, y \rightarrow p^{-}}\{[1+G(x, p)]-[1+G(x, y)][1+G(y, p)]\}$ $=\lim _{x, y \rightarrow p^{-}}\{G(x, p)-G(x, y)-G(y, p)-G(x, y) G(y, p)\}$, and

(2) $0=\lim _{x, y \rightarrow p^{-}}-\{[1-G(x, p)]-[1-G(x, y)][1-G(y, p)]\}$

Thus, $=\lim _{x, y \rightarrow p^{-}}\{-G(x, p)+G(x, y)+G(y, p)-G(x, y) G(y, p)\}$.

(3) $0=\lim _{x, y \rightarrow p^{-}}[G(x, p)-G(x, y)-G(y, p)]$, and

(4) $0=\lim _{x, y \rightarrow p^{-}} G(x, y) G(y, p)$.

Note that limits (1), (3) and (4) are the same as limits (2), (1) and (3), respectively, in Theorem 9 of a previous paper of the author [6]. It follows that $G\left(p^{-}, p\right)$ exists by the argument used in Theorem 9 [6]. Thus, it follows from (3) and the existence of $G\left(p^{-}, p\right)$ that $G\left(p^{-}, p^{-}\right)$exists and is zero. Therefore, since right limits can be treated similarly, $G \in O L^{14}$ on $[a, b]$. 
Lemma 5.3. If eacb of $a^{b}(1+G)$ and $\Pi^{b}(1-G)$ exists and is not zero, then $\int_{a}^{b} G^{2}$ exists.

Proof. Since each of $\Pi^{b}(1+G)$ and $\Pi^{b}(1-G)$ exists and is not zero, $\Pi^{b}\left(1-G^{2}\right)$ exists and is not zero. Thus, since $G$ is bounded on $[a, b]$ [Lemma 5.1], $-G^{2} \in O Q^{\circ}$ on $[a, b]$ [Lemma 1.3]. Since $G \in O L^{14}$ on [a, b] [Lemma 5.2], there exists a subdivision $\left\{x_{q}\right\}_{0}^{n}$ of $[a, b]$ such that if $1 \leq q \leq n$ and $x_{q-1}<x<$ $y<x_{q}$, then $|G(x, y)|<1$. Thus, since $-G^{2} \in O Q^{\circ}$ on $[a, b]$, it follows that $G^{2} \in O B^{\circ}$ on $\left[x_{q-1}, x_{q}\right]$ for $1 \leq q \leq n$. This is true because if $F$ is a function such that $0 \leq F \leq 1$ on an interval $[r, s]$ and $F \notin O B^{\circ}$ on $[r, s]$, then $-F \notin O Q^{\circ}$ on $[r, s]$. Therefore, $G^{2} \in O B^{\circ}$ on $[a, b]$. Lemma 1.4 implies that $-G^{2} \in O M^{\circ}$ on $[a, b]$. Hence, it follows from Lemma 2.1 that $\int_{a}^{b} G^{2}$ exists.

Lemma 5.4. If $\beta>0,|G|<1-\beta$ on $[a, b], \int_{a}^{b} G^{2}$ exists and $\Pi^{b}(1+G)$ exists and is not zero, then $\int_{a}^{b} G$ exists $[6$, Theorem 5$]$.

Theorem 5. If eacb of $\Pi^{b}(1+G)$ and $\Pi^{b}(1-G)$ exists and is not zero and $c$ is a number, then $c G \in O M^{\circ}$ and $O A^{\circ}$ on $[a, b]$.

Proof. Since $G \in O L^{14}$ on $[a, b]$ [Lemma 5.2], there exists a subdivision $D=\left\{x_{q}\right\}_{0}^{n}$ of $[a, b]$ ' such that if $1 \leq q \leq n$ and $x_{q-1}<x<y<x_{q}$, then $|G(x, y)|$ $<1 / 2$. Let $F$ be the function such that

(1) $F(x, y)=G(x, y)$ if $x \notin D$ and $y \notin D$, and

(2) $F(x, y)=0$ if $x \in D$ or $y \in D$.

Since $\int_{a}^{b} G^{2}$ exists [Lemma 5.3], $\int_{a}^{b} F^{2}$ also exists [Lemma 1.6]. Further, since $G \in O L^{14}$ on $[a, b]$ and $a^{b}(1+G)$ exists and is not zero, $\Pi^{b}(1+F)$ exists and is not zero [Lemma 1.7]. Therefore, $\int_{a}^{b} F$ exists [Lemma 5.4], and hence, $\int_{a}^{b} G$ exists [Lemma 1.6]. Thus, since $\int_{a}^{b} G^{2}$ exists and $\int_{a}^{b} G$ exists, it follows from Theorem 1 that $c G \in O M^{\circ}$ and $O A^{\circ}$ on $[a, b]$.

Theorem 6. If eacb of $a^{b}(1+G)$ and $\Pi^{b}(1-G)$ exists and is not zero, $H \in O L^{\circ}$ on $[a, b]$ and $n \geq 2$ is an integer, then $H G^{n} \in O M^{\circ}$ and $O A^{\circ}$ on $[a, b]$.

Proof. It follows as in Theorem 5 that $\int_{a}^{b} G^{2}$ exists and $\int_{a}^{b} G$ exists. Hence, Theorem 2 implies that $H G^{n} \in O M^{\circ}$ and $O A^{\circ}$ on $[a, b]$.

\section{BIBLIOGRAPHY}

1. W. D. L. Appling, Interval functions and real Hilbert spaces, Rend. Circ. Mat. Palermo (2) 11 (1962), 154-156. MR 27 \#4040.

2. W. P. Davis and J. A. Chatfield, Concerning product integrals and exponentials, Proc. Amer. Math. Soc. 25 (1970), 743-747. MR $42 \# 1970$. 
3. B. W. Helton, Integral equations and product integrals, Pacific J. Math. 16 (1966), 297-322. MR $32 \# 6167$.

4. - A product integral representation for a Gronwall inequality, Proc. Amer. Math. Soc. 23 (1969), 493-500. MR $40 \# 1562$.

5. J. C. Helton, Existence theorems for sum and product integrals, Proc. Amer. Math. Soc. 36 (1972), 407-413.

6. - Some interdependencies of sum and product integrals, Proc. Amer. Math. Soc. 37 (1973), 201-206.

7. - Product integrals, bounds and inverses, Texas J. Sci. 25 (1973) (to appear).

8. A. Kolmogoroff, Untersuchungen über den Integralbegriff, Math. Ann. 103 (19.30), $654-696$.

DEPARTMENT OF MATHEMATICS, ARIZONA STATE UNIVERSITY, TEMPE, ARIZONA 85281 